\title{
Commentary
}

Hemophagocytic lymphohistiocytosis (HLH) is a hyperinflammatory disorderresulting from immune dysfunction, reflecting either primary immune deficiency or acquired failure of normal immune homeostasis. Familial HLH includes autosomal recessive and X-linked disorders characterized by uncontrolled activation of $\mathrm{T}$ cells and macrophages and overproduction of inflammatory cytokines, secondary to defects in genes encoding proteins involved in granule-dependent cytolytic pathways. ${ }^{[1-3]}$ X-linked lymphoproliferative disease (XLP) is a primary immunodeficiency affecting approximately 1 to 3 per million live male births. Patients are generally healthy until facing a viral infection, such as Epstein-Barr virus. They may then develop fulminant infectious mononucleosis and die. XLP patients are also at increased risk of HLH, which may be triggered by assorted viruses. In older children and adults, HLH is associated more often with infections, malignancies, autoimmune diseases, and acquired immune deficiencies.

HLH, macrophage activation syndrome, sepsis, and systemic inflammatory response syndrome are different clinical entities that probably represent a common immunopathological state termed cytokine storm. These entities share as physiopathogenic mechanism the presence of an impaired and inefficacious function of the NK and T cytotoxic cells that leads to an ineffective and uncontrolled immune response, inducing cellular damage, multiorganic failure with macrophage proliferation and hemophagocytosis. ${ }^{[4]}$ These conditions may be clinically indistinguishable; all include massive inflammatory response, elevated serum cytokine levels, multi-organ involvement, hemophagocytic macrophages, and often death. Tissues of hematopoietic and lymphoid function are directly involved; other organs are secondarily damaged by circulating cytokines and chemokines. ${ }^{[1,2]}$ Although an early diagnosis is crucial to decrease mortality, the definitive diagnosis is often challenging because of the lack of specificity of currently accepted diagnostic criteria and the absence of confirmatory gold standards. ${ }^{[5]}$

The reported central nervous system (CNS) involvement, independently of the type of HLH, is variable and up to $70 \%$ of patients can show CNS compromise in any time of the disease course. Despite the relative common compromise of the CNS, the literature describing neuroimaging findings is scarce. In present article, the authors present the case of a 4.5-year-old male patient diagnosed with XLP in the context of an EBV infection and HLH with fatal course despite aggressive treatment. ${ }^{[6]}$ The progression of the depicted lesions on the initial presentation of the brain MRI with respect to the repeated MRI a month later correlated with the fatal course of the patient. Additionally, the MR spectroscopy findings were consistent with an inflammatory necrotic damage of the brain. As reported by Rego et al., the presence of CNS involvement at diagnosis is a poor prognostic sign. He described the neuroradiological findings, clinical data, and treatment outcome in 12 patients with genetic HLH and CNS complications. Neuroimaging was important in identifying CNS involvement, monitoring treatment responses, and detecting treatment complications. ${ }^{[7]}$

The main objective of the different therapeutic options-commonly combinations of steroids and 
chemotherapy- is the suppression of the uncontrolled immune response. ${ }^{[4]}$ Although, the clinical and neuroradiological findings are not distinguishable between the familial and acquired forms of HLH and the neuroradiological findings in HLH are not sufficiently specific to achieve the diagnosis. The CNS compromise may help to change or intensify the therapeutic efforts in order to optimize the drugs concentrations in the CNS. Most of the therapeutic failures are due to the inadequate drugs diffusion through the blood brain barrier.

\section{Carolina Mahuad}

Department of Internal Medicine, PhD in Biomedical Sciences, Specialist in Internal Medicine and Hematology, Hematology Service, German Hospital, Buenos Aires, Argentina

Address for correspondence: Dr. Carolina Mahuad, Department of Internal Medicine, Hematology Service, German Hospital, Pueyrredon 1640, 1118 CABA, Argentina. Email: c.mahuad@hospitalaleman.com

\section{References}

1. Usmani GN, Woda BA, Newburger PE. Advances in understanding the pathogenesis of HLH. Br J Haematol 2013;161:609-22.

2. Sperl D, Benesch M, Urban C, Lackner H, Sovinz P, Speicher MR, et al.
Fatal EBV infection and variable clinical manifestations in an XLP-1 pedigree -rapid diagnosis of primary immunodeficiencies may save lives. Klin Padiatr 2012;224:386-9.

3. Booth C, Gilmour KC, Veys P, Gennery AR, Slatter MA, Chapel H, et al. $\mathrm{X}$-linked lymphoproliferative disease due to SAP/SH2D1A deficiency: A multicenter study on the manifestations, management and outcome of the disease. Blood 2011;117:53-62.

4. Mahuad CV, Garate GM, Vicente Reparaz Mde L, Casali C, Del Olmo M, Bolgiani A. Secondary hemophagocytic syndrome due to recurrent infections in a severely burned patient. Medicine (B Aires) 2013;73:255-8.

5. Rosado FG, Kim AS. Hemophagocytic lymphohistiocytosis: An update on diagnosis and pathogenesis. Am J Clin Pathol 2013;139:713-27.

6. Sankararaman S, Riel-Romero RM, Jeroudi M, Gonzalez-Toledo E. Epstein-Barr virus induced hemophagocytic lymphohistiocytosis in X-linked lymphoproliferative disease. J Neurosci Rural Pract 2014;5:171-4.

7. Rego I, Severino M, Micalizzi C, Faraci M, Pende D, Dufour C, et al. Neuroradiologic findings and follow-up with magnetic resonance imaging of the genetic forms of haemophagocyticlymphohistiocytosis with CNS involvement. Pediatr Blood Cancer 2012;58:810-4.

\begin{tabular}{|l|l|}
\hline \multicolumn{2}{|c|}{ Access this article online } \\
\hline Quick Response Code: & \\
\hline & Website: \\
\hline
\end{tabular}

